\title{
Diagnostic and prognostic role of Ki-67 and cytokeratin-5 expression in BPH and carcinoma prostate
}

\author{
Bakna $\mathbf{M}^{1}$, Malik $\mathbf{R}^{2}$, Jain $\mathbf{P}^{3}$, Jain $\mathbf{R}^{4}$, Jain $\mathbf{A}^{5}$ \\ ${ }^{1}$ Dr. Minu Bakna, M.B.B.S., Resident, Department of Pathology, ${ }^{2}$ Dr. Reeni Malik, Professor, Department of Pathology, \\ ${ }^{3}$ Dr. Pramila Jain, Associate Professor, Department of Pathology, ${ }^{4}$ Dr. Rashmi Jain, Resident, Department of Pathology, \\ ${ }^{5}$ Dr. Ankit Jain, Resident, Department of Anatomy, all authors affiliated with Gandhi Medical College, Bhopal (M.P.), \\ India.
}

Address for Correspondence Dr. Minu Bakna, Institute: Department of Pathology, Gandhi Medical College, Bhopal (M.P.), Postal Address: Dr. Minu Bakna C/O Mr. R.S.Bakna, Behind Patali Hanuman, Tansen Road, Birla Nagar, Gwalior, (M.P.), E-mail: minubakna@gmail.com

\begin{abstract}
Background: Cytokeratin-5 which stains the basal cell layer of the of the prostatic glands and Ki-67 which is a proliferation marker and stains the proliferative population of the lesion has been employed as markers of two different categories viz. basal cell marker and proliferative markers respectively in various studies. Aim of this study was to evaluate the utility of cytokeratin-5 and Ki-67 in differentiating and diagnosing the benign lesion like Benign prostatic hyperplasia and malignant lesion i.e. Carcinoma Prostate. Material and Methods: 90 cases of prostatic adenocarcinoma, $\mathrm{BPH}$ and prostatic inflammatory conditions were taken. Immunohistochemical staining for Ki67 and CK-5 was performed along with appropriate positive controls for each marker. Ki67 scoring classified as Negative, Low grade \& High grade. Cytokeratin-5 scoring classified as Diffuse, Focal \& Negative. Results: Cytokeratin-5 expression was found positive in $96.71 \%$ cases of BPH. Out of these $78.68 \%$ cases were diffusely positive and $18.03 \%$ cases were focal positive. Expression of Cytokeratin-5 in Carcinoma Prostate was 40\%, 6.66\% and 0\% in well, moderate and poorly differentiated carcinomas respectively, whereas no expression of the marker in $60 \%, 93.33 \%$ and $100 \%$ cases of well, moderate and poorly differentiated carcinomas respectively. Ki-67 showed low grade expression in mere $8.20 \%$ cases of BPH. In Carcinoma Prostate Ki67 expression was shown by $60 \%, 79 \%$ and $100 \%$ cases of well, moderate and poorly differentiated carcinomas respectively. Conclusion: Diffuse Cytokeratin-5 expression was found in majority of BPH cases, while maximum Carcinoma prostate cases were negative. Ki-67 expression was found positively correlated with increasing Gleason's grade in carcinoma prostate, while expression in benign lesion was not significant.
\end{abstract}

Keywords: Ki-67, Cytokeratin-5, BPH, Carcinoma prostate.

\section{Introduction}

As the life expectancy has improved in last few decades, there has been an associated increase of geriatric population worldwide which has got spectrum of ailments, one of the commonest ailments being the prostatic enlargement [1]. The two most common causes of prostatic enlargement are benign prostatic hyperplasia and carcinoma prostate and in India BPH attributes to $93.3 \%$ and prostatic cancer ranks $5^{\text {th }}$ in incidence and $4^{\text {th }}$ in cancer mortality for men [2]. As these diseases share common pathogenic mechanism

Manuscript received $26^{\text {th }}$ July 2016

Reviewed: $7^{\text {th }}$ August 2016

Author Corrected: $18^{\text {th }}$ August 2016

Accepted for Publication $31^{\text {st }}$ August 2016 and a similar clinical picture, a pathologist may face great complications in the process of early diagnosis of prostate cancer. So for the definitive diagnosis histopathological and immunohisto-chemical interpretation of transrectal needle core biopsy and radical prostatectomy specimens has proven to be very important and decisive [3].

Prostate carcinoma is a complex and multistep disease, and no single morphologic feature can be specific for cancer and many benign conditions can mimic prostate cancer. So clinical and pathological staging along with the prediction of degree of aggressiveness of the disease 
(which is done by examining the tissues obtained from the patients), influence the choice of therapeutic intervention for the patients of carcinoma prostate. Therefore accurate prediction of the behavior of individual's tumor is a major challenge [4].

Cytokeratin-5 which can differentiate the benign lesions from the malignant lesions, and Ki-67 which can assess the proliferative potential of a lesion and the clinical course of the malignant disease were used in the present immunohistochemical study [3].

$\mathrm{Ki}$-67- As this is a proliferation marker, its expression is influenced by the cell proliferation activity in a particular tissue. During the interphase, the expression of this antigen is restricted only within the nucleus, whereas in mitosis, the majority of this protein gets translocated to the surface of the chromosomes. As Ki67 protein is expressed during all the active phases of the cell cycle (G1,S,G2 and mitosis), but is not expressed in the G0 phase, this property has made it a reliable marker for estimating the growth fraction of a determined cell population (normal or tumoral)[5]. The Ki-67 positive tumor cell fraction has been correlated with the clinical course the disease, chances of recurrence and the survival of patient in prostate cancer, suggesting that $\mathrm{Ki}-67$ can be used as a prognostic factor in the monitoring of these patients [5].

The Ki67-positive tumor cell fraction (the Ki67 labeling index/ Ki67-LI) is correlated with the clinical course of the disease, including the cases of Carcinoma prostate, where cell proliferation evaluated by Ki-67 expression is found to increase from the cases of localized Carcinoma Prostate to the metastatic cases [5]. A high Ki67 LI is independently associated with seminal vesicle infiltration and high postoperative Gleason's score. More importantly, the Ki67 LI can also predict the chances of biochemical recurrence, particularly in the cases where only a small fraction of tumor biopsy was sent. The Ki67 LI is also be a valid predictor of recurrence-free survival after radical prostatectomy $[6,7]$.

Cytokeratin-5- The cytokeratin filaments are composed of proteins which are related to, but not identical with, epidermal $\alpha$ keratins, are a complex family of many different polypeptides and have apparent molecular weights from 40,000 to $68,000 \mathrm{kDa}$. So the pattern of cytokeratin component of a particular epithelium or epithelial cell can therefore characterize the type of the epithelium [8].
There are 23 subtypes of cytokeratin (CK) intermediate filaments. These have different molecular weights and they demonstrate differential expression in different cell types and different tumors. Monoclonal antibodies (MoAb) to specific CK subtypes have been used to classify tumors according to their site of origin [9].

Cytokeratins (CKs) are subdivided into two subclasses [10]:-

\section{a. Type I (CK9-CK23) \\ b. Type II (CK1-CK8)}

In the normal and pre-invasive human prostate glandular tissue, the luminal epithelium is separated from the stroma by a layer of basal cells and a basement membrane. Basal cells encircles the entire luminal epithelium of the duct and acinic system in a continuous fashion, and are absent in cancerous glands. So the immunohistochemical identification of basal cell layer is considered a reliable criterion for differentiating the non-invasive from the invasive lesions of the prostate. For the diagnosis of Carcinoma prostate, several basal cell markers, such as high-molecular weight cytokeratin (34ßE12), cytokeratin5/6 (keratin 5/6), p53 and bcl-2 have been implicated [11].

The usefulness of Ck-5 as a "negative" marker has been documented previously. CK-5 is an excellent, consistent, and reliable basal cell marker for the assessment of routine prostate specimens.CK-5 exhibits excellent specificity for prostate cancer, which uniformly lacks CK-5 staining (100\% negative). Cases of BPH show continuous, intact and circumferential staining while PIN cases shows mostly fragmented basal cell staining for CK5/6, sometimes restricted to only rare positive basal cells [12].

Cytokeratin-5, normally expressed by complex epithelium is a marker mesothelial cells and mesothelioma, as well as pancreatic bile duct and breast carcinomas. Antibody of cytokeratin-5 also reacts with prostate gland basal cells. Overall sensitivity, specificity and diagnostic utility of cytokeratin- 5 in prostate biopsies is said to be similar to those of 34 $\beta \mathrm{E} 12$ [11].

This study was performed to assess the expression of Ki-67 and Cytokeratin-5 in the cases of benign hyperplasia of Prostate \& Carcinoma Prostate, so as to assess their utility as predictive and prognostic indicators for these diseases. 


\section{Materials and Methods}

This study was a retrospective study conducted in the Department of Pathology, Gandhi Medical College, Bhopal, after taking ethical committee clearance. 90 cases of prostatic adenocarcinoma, BPH and prostatic inflammatory conditions were taken. Carcinomas other than prostatic adenocarcinomas, insufficient biopsies and autolyzed specimens were excluded.

Demographical informations viz. age, details of tumor, histopathological diagnosis with Gleason's Grade of the tumor at the time of diagnosis were noted from the departmental records. Paraffin blocks of all selected cases were retrieved from Department and checked, processed and stained by haematoxylin and eosin staining and reviewed. Grading was done according to Gleason's Grade in malignant cases. After confirming and noting the diagnosis immunohistochemical staining for Ki67 and CK-5 was performed along with respective positive controls.

3-4 mm thick sections were cut and gently lowered on surface of water bath at $45^{\circ} \mathrm{C}$ and were spread wrinkle free on to the slides coated with $0.1 \%$ poly L-lysine for 15 minutes at $37^{\circ} \mathrm{C}$ and air dried. Then the slides were kept on hot plate at $60^{\circ} \mathrm{C}$ for baking for 30 minutes. Dewaxing was done by treating the slides in xylene followed by rehydration in absolute alcohol, 90\% alcohol and $70 \%$ alcohol. For antigen retrieval slides were put in preheated pressure cooker having citrate buffer, than boiled and allowed to cool naturally. To block the endogenous enzyme activity hydrogen peroxide was added in a moist chamber at room temperature. Then primary antibody was added for 1-2 hrs in moist chamber at room temperature.
Enhancement of primary antibody was done by adding antibody enhancer (super enhancer) and incubated in moist chamber for 20min. The peroxidase antiperoxidase method was followed for secondary staining. DAB was used for coloring the antigen-antibody complex. (Primary and secondary antibodies were obtained from Biogenix USA). This was followed by counterstaining with hematoxylin $[13,14]$.

Ki67 scoring[15]: Sections were examined under 400x magnification\& distinct nuclear staining was taken as positive .500 cells were counted in the area of maximum $\mathrm{Ki}-67$ positivity. The percentage of cells positive for Ki67 was scored semi-quantitatively, according to the number of labeled cells observed as-

Negative $=$ no staining $;$ Low grade $=<15 \%$ \& High grade $=>15 \%$

Cytokeratin-5 scoring [12]:- Sections were examined under 400x magnification. Distinct cytoplasmic staining was taken as positive. It was scored semiquantitatively:-

Diffuse -all glands positive; Focal - some glands or gland portions positive \& Negative- no glands stained positive.

The results of the study were statistically analyzed using the Statistical Package for the Social Sciences (SPSS) version 20 (IBM Corp. SPSS statistics, in Armonk NY) for windows. Data were expressed as mean \pm SD for the quantitative variables, numbers, and percentage. Comparison between multiple groups were made using student $t$-test for quantitative data and Chisquare test for qualitative data. A value of $\mathrm{P}<0.05$ was taken as significant and $\mathrm{P}<0.01$ was taken as highly significant.

\section{Results}

We observed that the maximum number of cases of BPH i.e. 31 cases $(50.81 \%)$ and also of the carcinoma prostate i.e. 13 cases $(44.82 \%)$ cases belonged to the age group of 61-70 years [Table-1].

Table-1: Age wise distribution of prostatic lesions (n=90).

\begin{tabular}{|c|c|c|c|c|}
\hline \multirow{2}{*}{ Age (in years) } & \multicolumn{2}{|c|}{ Benign Prostatic Hyperplasia (n=61) } & \multicolumn{2}{c|}{ Prostate Carcinoma (n=29) } \\
\cline { 2 - 5 } & No. of cases & No. of cases & \% \\
\hline$<50$ & 4 & $6.55 \%$ & 1 & $2.44 \%$ \\
\hline $\mathbf{5 1 - 6 0}$ & 19 & $31.11 \%$ & 6 & $44.82 \%$ \\
\hline $\mathbf{6 1 - 7 0}$ & 31 & $50.81 \%$ & 13 & $31.03 \%$ \\
\hline$>70$ & 7 & $11.47 \%$ & 9 & \\
\hline
\end{tabular}


Out of the 29 cases of carcinoma prostate, most of the cases i.e. $27.58 \%$ cases belonged to Gleason's score of $7 \&$ the maximum percentage of cases belonging to the moderately differentiated Carcinoma Prostate (Gleason score 5, 6 \& 7) was $51.71 \%$ [Table-2].

Table-2: Distribution of cases of Prostate Carcinoma under each Gleason score $(\mathbf{n}=29)$.

\begin{tabular}{|c|c|c|c|}
\hline $\begin{array}{c}\text { Differentiation of } \\
\text { Prostatic carcinoma }\end{array}$ & Gleason score & No. of Cases & \% \\
\hline Well & $2-4$ & 5 & $17.24 \%$ \\
\hline Moderate & $5-7$ & 15 & $51.72 \%$ \\
\hline Poor & $8-10$ & 9 & $31.04 \%$ \\
\hline
\end{tabular}

Table-3: Expression of Cytokeratin-5 (CK-5) in Prostatic lesions (n=90).

\begin{tabular}{|c|c|c|c|c|c|c|}
\hline \multirow{3}{*}{\multicolumn{2}{|c|}{ Prostatic lesions }} & \multicolumn{3}{|c|}{ CK-5 expression } & \multirow[t]{3}{*}{ Total } & \multirow[t]{3}{*}{ P value* } \\
\hline & & \multicolumn{2}{|c|}{ Positive } & \multirow[t]{2}{*}{ Negative } & & \\
\hline & & Diffuse & Focal & & & \\
\hline \multicolumn{2}{|c|}{$\begin{array}{l}\text { Benign Prostatic Hyperplasia } \\
\qquad(n=61)\end{array}$} & $\begin{array}{c}48 \\
(78.68 \%)\end{array}$ & $\begin{array}{c}11 \\
(18.03 \%)\end{array}$ & $2(3.27 \%)$ & $61(100 \%)$ & \\
\hline \multirow{3}{*}{$\begin{array}{c}\text { Prostate } \\
\text { carcinoma } \\
(\mathbf{n}=\mathbf{2 9})\end{array}$} & Well & \multicolumn{2}{|c|}{$2(40 \%)$} & $3(60 \%)$ & $5(100 \%)$ & \multirow[t]{3}{*}{$.049^{\mathrm{a}}$} \\
\hline & Moderate & \multicolumn{2}{|c|}{$1(6.66 \%)$} & $14(93.33 \%)$ & $15(100 \%)$ & \\
\hline & Poor & \multicolumn{2}{|c|}{0} & $(100 \%)$ & $9(100 \%)$ & \\
\hline
\end{tabular}

*Statistical analysis of data was done by using Chi-square test. $\mathrm{P}$ value $<0.05$ was considered significant. a. Significant.

Table-4: Expression of Ki-67 in Prostatic lesions (n=90).

\begin{tabular}{|c|c|c|c|c|c|c|}
\hline \multirow{2}{*}{ Prostatic lesions } & \multicolumn{3}{|c|}{ Ki-67 expression } & \multirow{2}{*}{ Total } & P value* \\
\cline { 2 - 6 } & Negative & low & high & & \\
\hline \multirow{2}{*}{$\begin{array}{c}\text { Benign Prostatic Hyperplasia } \\
(\mathbf{n}=\mathbf{6 1})\end{array}$} & $56(91.8 \%)$ & $5(8.2 \%)$ & 0 & $61(100 \%)$ & \\
\hline \multirow{2}{*}{$\begin{array}{c}\text { Prostate } \\
\text { carcinoma } \\
(\mathbf{n = 2 9})\end{array}$} & Well & $2(40 \%)$ & $3(60 \%)$ & 0 & $5(100 \%)$ & $.072^{\#}$ \\
\cline { 2 - 6 } & Moderate & $3(20 \%)$ & $4(26.66 \%)$ & $8(53.33 \%)$ & $15(100 \%)$ & \\
\cline { 2 - 6 } & Poor & 0 & $2(22.22 \%)$ & $7(77.78 \%)$ & $9(100 \%)$ & \\
\hline
\end{tabular}

*Statistical analysis of data was done by using Chi-square test. P value $<0.05$ was considered significant. \# statistically not significant.

The basal cell marker, Cytokeratin-5 expression was found positive in $96.71 \%$ cases of BPH. Out of these $78.68 \%$ cases were diffusely positive and $18.03 \%$ cases were showing focal positivity for the marker. Positive expression of Cytokeratin-5 in Carcinoma Prostate was 40\%, 6.66\% and $0 \%$ in well, moderate and poorly differentiated carcinomas respectively, whereas there was no expression of the marker in $60 \%, 93.33 \%$ and $100 \%$ cases of well, moderate and poorly differentiated carcinomas respectively. Thus relationship of Cytokeratin-5 expression was found to be related inversely with the grading of the Prostate carcinoma [Table-3].

Proliferation marker Ki-67 showed only low grade expression in mere 8.20\% cases of BPH. In Carcinoma Prostate, positive expression of Ki-67 was shown by $60 \%, 79 \%$ and $100 \%$ cases of well, moderate and poorly differentiated carcinomas respectively. In well differentiated carcinomas all the positive cases were showing low grade expression. In moderately differentiated carcinomas $26.66 \%$ cases were showing low grade and $53.33 \%$ cases were showing high grade expression. In poorly differentiated carcinomas $22.22 \%$ cases were showing low grade and $77.78 \%$ cases were showing high grade expression [Table-4] 


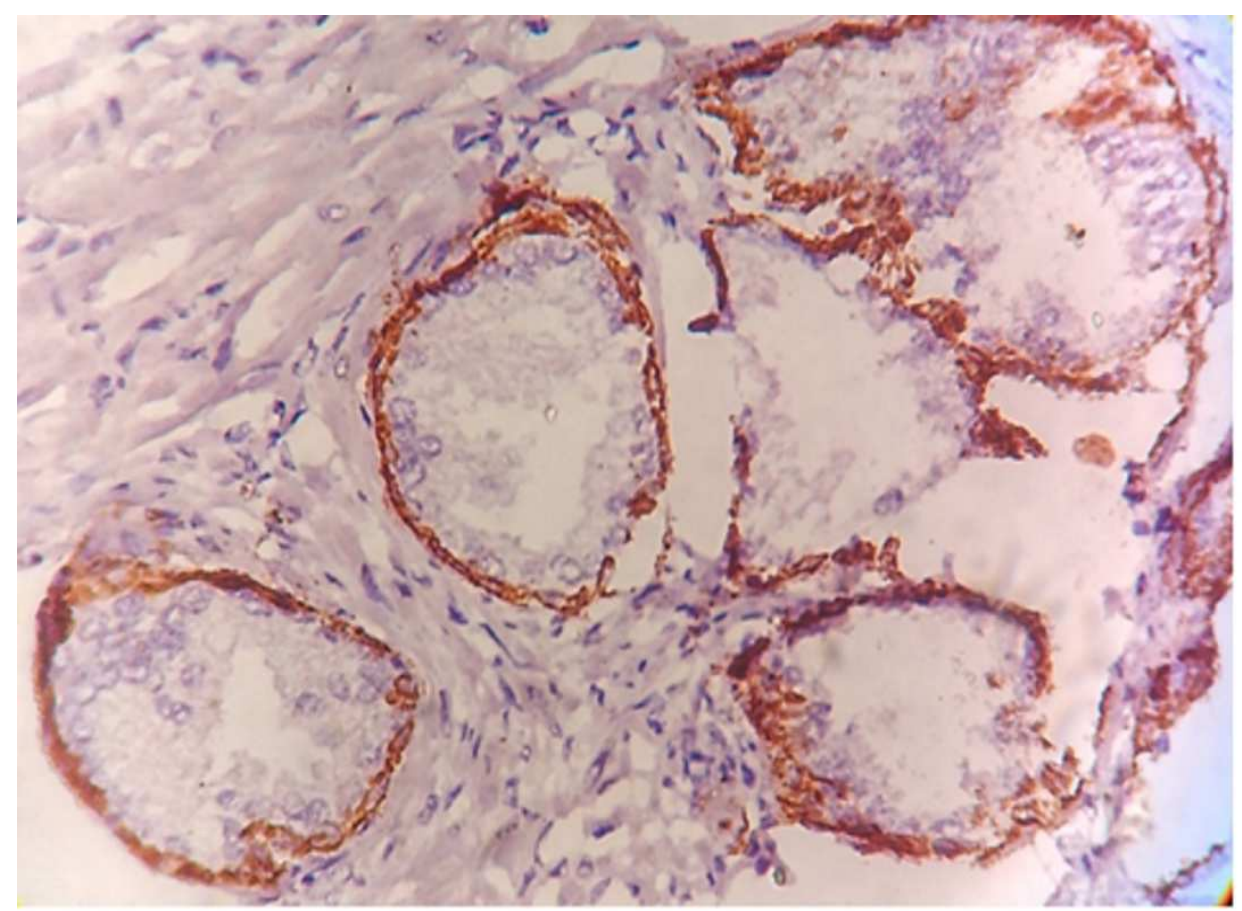

Image-1: IHC STAIN SLIDE OF BPH SHOWING DIFFUSE CONTINUOUS CYTOKERATIN-5 POSITIVITY

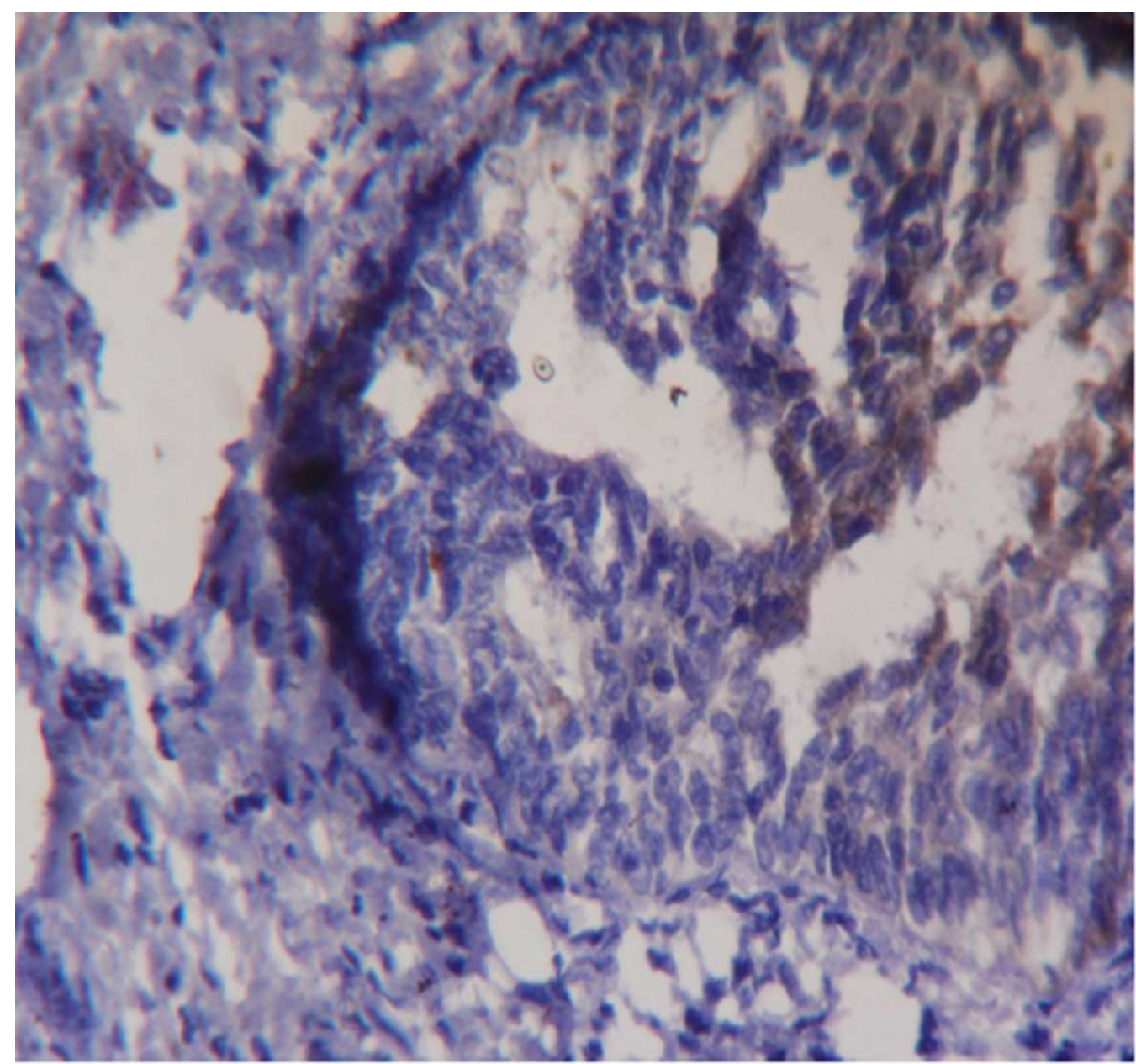

Image- 2: IHC stain slide of carcinoma prostate showing negative cytokeratin-5 staining in PCa 


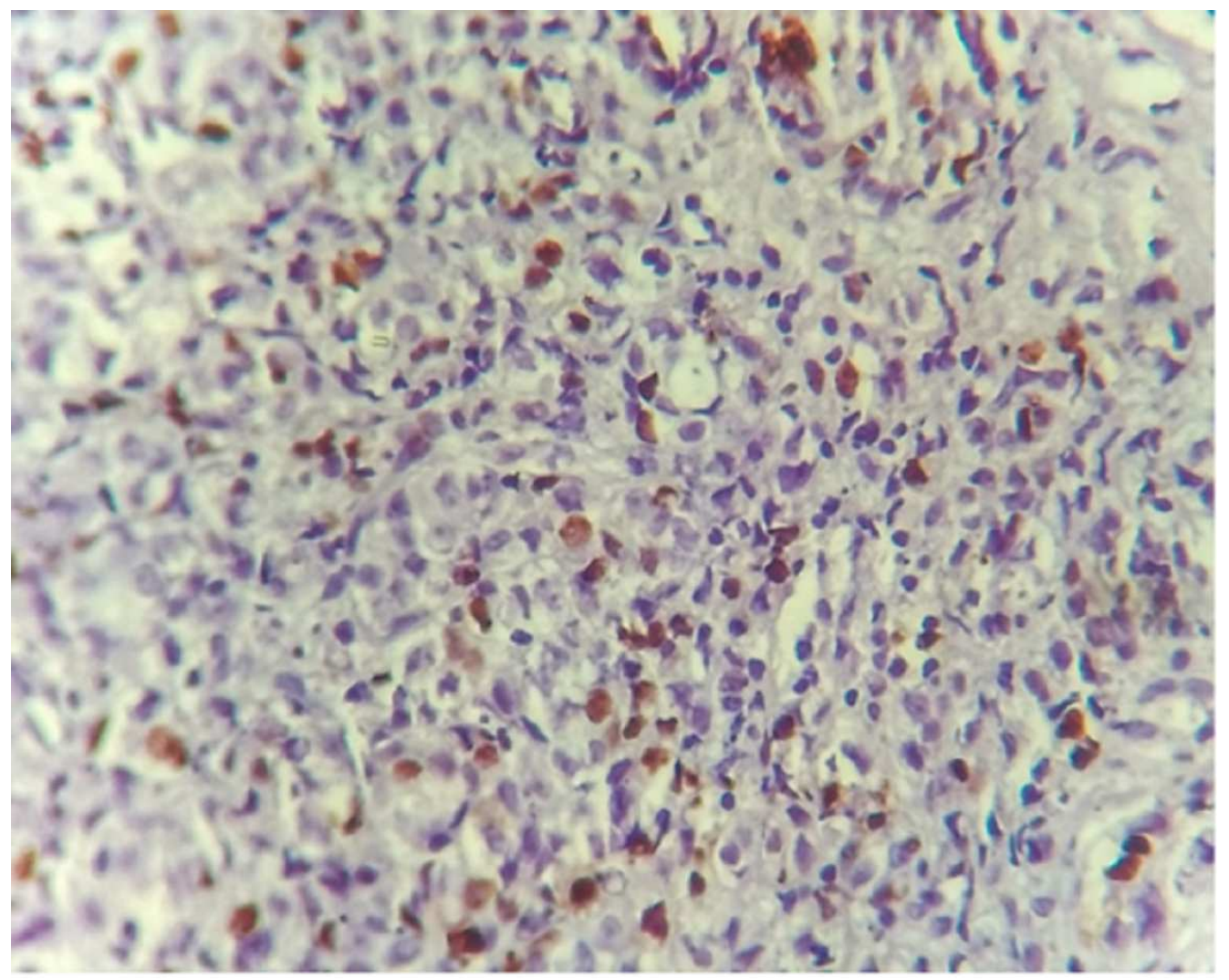

Image-3: IHC stain slide of carcinoma prostate showing high grade ki67 positivity in moderately differentiated carcinoma prostate

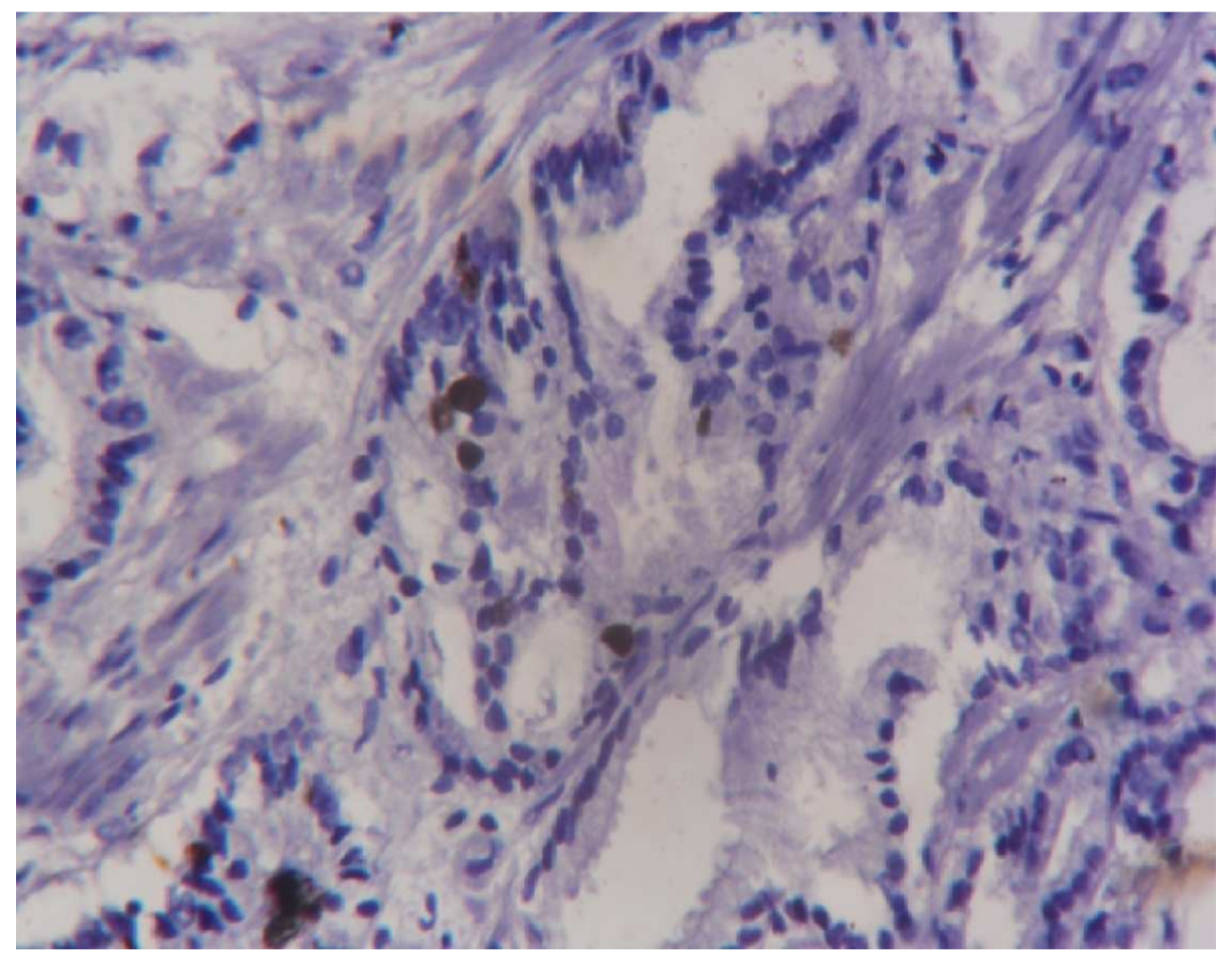

Image 4: IHC stain slide of carcinoma prostate showing low ki-67 positivity $(<15 \%)$ in well differentiated carcinoma prostate 


\section{Discussion}

Carcinoma prostate is biologically very heterogenous, and its clinical course may vary from indolent entity to a rapidly growing tumor with early metastasis, attributable to the genetic alterations. New biologic markers associated with a tumor's aggressiveness may provide significant prognostic information [16]. Extensive work in field of IHC have established the role of Cytokeratin-5 and Ki-67as important diagnostic and prognostic markers for the differentiation between and the diagnosis of BPH and Carcinoma Prostate. So these markers of carcinogenesis have a significant role in detecting \& treating Carcinoma Prostate as they can supplement information on standard prognostic and predictive indicators viz. clinical staging, PSA level and Gleason's score. In order to make a comparative evaluation of processes known to be crucial for carciongenesis, like cell proliferation and loss of basal layer, immunohistochemical staining of their representative proteins in the tissues derived from the patient is necessary[17].

The proliferation rate, a feature that has been proven to be an unfavourable prognostic factor in many cancers was evaluated by Ki-67 protein by many authors. Stapleton et al in his study found that increased Ki-67 expression was correlated significantly with advanced clinical stage of prostate carcinoma. He also found that in the patients with metastatic disease, increased Ki-67 tissue levels predicted a shorter survival time and increased chances of disease recurrence[16]. Makarewicz et al found a significant relationship between the expression of $\mathrm{Ki}-67$ with increasing Gleason's score confirming the role of proliferation in carcinogenesis and disease progression.

He found that an increasing proliferation rate seems to promote a lower degree of differentiation in cancer cells and also confirmed that a high proliferative index in Prostate Carcinoma is associated with radiotherapy failure, a higher risk of metastasis and a shorter recurrence free survival [17].In our study we found that $91 \%$ of BPH cases were negative for Ki-67 staining, going with the fact that BPH is a disease associated with decreased rate of apoptosis rather than increased proliferation [Table-4]. On the other hand, Ki-67 expression in Carcinoma prostate was positively correlated (although not statistically significant) with increasing Gleason's score, similar to the findings of the above authors stating the role of proliferation in carcinogenesis, disease progression, prediction of survival time and disease recurrence [Table-4].

Staining for basal cell marker has been a yard stick in differentiating the benign lesions from the malignant ones, and many basal cell markers e.g. AMACR, $34 \beta E 12$, p63 etc. were studied by different authors. Trpkov K. et al studied the Cytokeratin-5 and AMACR staining and found that Cytokeratin-5 was more specific as basal cell marker as compared to the later. The complete lack of Cytokeratin staining, indicating the absence of basal cells in a suspicious focus, lends the diagnosis of Prostate Cancer[18]. Goldstein $S$ et al observed that there can be sparse cytokeratin immunoreactivity in intermediate Gleason's score adenocarcinomas [19]. This is consistent with our results in which 3 of the total 29 cases of carcinoma prostate belonging to the well and intermediate differentiation was found to show some focal positivity with the marker. Adisa J.O. et al in his study has also stated that the Cytokeratin-5 expression is negatively correlated with Carcinoma Prostate and positively correlated in BPH cases, supporting our study [20].

\section{Conclusion}

We conclude that $\mathrm{Ki}-67$ as a marker of proliferation is a very reliable biological marker of Prostate Carcinoma and may be useful in differentiating it from BPH. It is upregulated in malignant lesions as compared to the benign lesions and a strong relationship with Gleason's grading was noticed. Ki-67 is also a strong predictor of poor prognosis, disease free survival and chances of recurrence after radical prostatectomy.

Cytokeratin-5 being an excellent marker for the demonstration of the basal layer of the prostatic glands shows a very useful potential in reaching to a firm diagnosis in cases of a doubtful foci in tissue biopsies, benign mimickers and very small biopsies. Both these markers complement each other, as cytokeratin indicate about the integrity of the glandular structure while $\mathrm{Ki}$ 67 indicating about the proliferative activity of the cells. Because the expression of these markers is easily estimated by immunohistochemical method, it may be applicable in everyday practice as an additional clinical tool and a prognostic factor.

Funding: Nil, Conflict of interest: None initiated, Permission from IRB: Yes 


\section{References}

1. Talukdar SI, Roy MK, Azam MS, Huq MH, Haque MA, Saleh AF. Histopathological patterns of prostate specimens in Mymensingh. Dinajpur Med Col J. 2008;1(2);29-32

2. Mathur SK, Gupta S, Marwah N, Narula A, Singh S, Arora B. Significance of mucin stain in differentiating benign and malignant lesions of prostate. Indian journal of pathology \& microbiology. 2003 Oct;46(4): 593-5.

3. Popkov VM, Maslyakova GN, Voronina ES. Immunohistochemical characteristics in diagnostics of prostate diseases. Russian Open Medical Journal. 2013; 2(1):0109.

4. Wood DP, Honn KV, editors. The oncobiology of the prostate. Elsevier; 2000 Dec 20.

5. Munoz E, Gomez F, Paz JI, Casado I. Ki-67 immunolabeling in pre-malignant lesions and carcinoma of the prostate. Histological correlation and prognostic evaluation. European Journal of Histochemistry. 2009 Jun 26;47(2):123-8

6. Sequeiros T, García M, Montes M, Oliván M, Rigau M, Colás E, de Torres I, Morote J, Reventós J, Doll A. Molecular markers for prostate cancer in formalin-fixed paraffin- embedded tissues. Bio Med research international. 2013 Nov 25;2013.

7. Verma R, Gupta V, Singh J, Verma M, Gupta G, Gupta S, Sen R, Ralli M. Significance of p53 and ki-67 expression in prostate cancer. Urology annals. 2015 Oct;7(4):488.

8. Moll R, Franke WW, Schiller DL, Geiger B, Krepler R. The catalog of human cytokeratins: patterns of expression in normal epithelia, tumors and cultured cells. cell. 1982 Nov 30;31(1):11-24.

9.Varadhachary GR, Abbruzzese JL, Lenzi R. Diagnostic strategies for unknown primary cancer. Cancer. 2004 May 1;100(9):1776-85.

10. Kanaji N, Bandoh S, Fujita J, Ishii T, Ishida T, Kubo A. Compensation of type I and type II cytokeratin pools in lung cancer. Lung Cancer. 2007 Mar 31;55(3):295-302.
11. Boran C, Kandirali E, Yilmaz F, Serin E, Akyol M. Reliability of the $34 \beta E 12$, keratin $5 / 6$, p63, bcl-2, and AMACR in the diagnosis of prostate carcinoma. InUrologic Oncology: Seminars and Original Investigations 2011 Dec 31 (Vol. 29, No. 6, pp. 614623). Elsevier

12. Trpkov K, Bartczak-McKay J, Yilmaz A. Usefulness of cytokeratin 5/6 and AMACR applied as double sequential immunostains for diagnostic assessment of problematic prostate specimens. American journal of clinical pathology. 2009 Aug 1;132 (2):211-20.

13. Diagnostic Immunohistochemistry: Theranostic and Genomic Applications: 4th Edition, David J Dabbs. Immune- histochemistry staining Biogenx Immunohistochemistry kit manual.

14. Madani SH, Ameli S, Khazaei S, Kanani M, Izadi B. Frequency of Ki-67 (MIB-1) and P53 expressions among patients with prostate cancer. Indian Journal of Pathology and Microbiology. 2011 Oct 1;54(4):688.

15. Stapleton AM, Zbell P, Kattan MW, Yang G, Wheeler TM, Scardino PT, Thompson TC. Assessment of the biologic markers p53, ki-67, and apoptotic index as predictive indicators of prostate carcinoma recurrence after surgery. Cancer. 1998 Jan 1;82(1): 168-75.

16. Makarewicz R, Zyromska A, Andrusewicz H. Comparative analysis of biological profiles of Comparative analysis of biological profiles of Comparative analysis of biological profiles of benign prostate hyperplasia and prostate cancer as benign prostate hyperplasia and prostate cancer as potential diagnostic, prognostic and predictive potential diagnostic, prognostic and predictive indicators. CPolish Society for Histochemistry and Cytochemistry Folia Histochem Cytobiol. 2011 10.5603/FHC. 2011.0064 Vol. 49, No. 3, 2011 pp. 452-457.

17. Trpkov K, Bartczak-McKay J, Yilmaz A. Usefulness of cytokeratin 5/6 and AMACR applied as double sequential immunostains for diagnostic assessment of problematic prostate specimens. American journal of clinical pathology. 2009 Aug $1 ; 132(2): 211-20$. 
18. Goldstein NS. Immunophenotypic characterization of 225 prostate adenocarcinomas with intermediate or high Gleason scores. American journal of clinical pathology. 2002 Mar 1;117(3):471-7.
19. Adisa JO, Egbujo EC, Ibrahim B, Musa B, Madukwe J. Expression of some selected cytokeratins and Ki67 protein in prostatic tumor: can these be used as tumor markers.The Pan African Medical Journal. 2015;20:46. doi:10.11604/pamj.2015.20.46.3926.

\section{How to cite this article?}

Bakna M, Malik R, Jain P, Jain R, Jain A. Diagnostic and prognostic role of Ki-67 and cytokeratin-5 expression in BPH and carcinoma prostate. Int J Med Res Rev 2016;4(8):1484-1492.doi:10.17511/ijmrr.2016.i08.32. 